\title{
Care Plan for Reducing Stigma in Cancer Disease
}

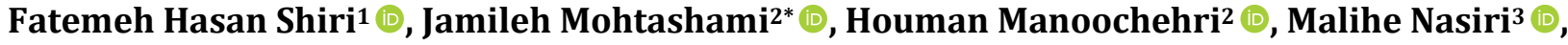 \\ Camelia Rohani2
}

\begin{abstract}
${ }^{1}$ Department of Anesthesiology, Faculty of Paramedical, Kashan University of Medical sciences, Kashan, Iran ${ }^{2}$ School of Nursing \& Midwifery, Shahid Beheshti University of Medical Sciences, Tehran, Iran ${ }^{3}$ Department of Biostatistics, School of Nursing \& Midwifery, Shahid Beheshti University of Medical Sciences, Tehran, Iran Email: Iran.hasanshiri7@gmail.com, ^J_mohtashami@sbmu.ac.ir,dr.houmanmanoochehri@gmail.com, malihenasiri@gmail.com,camelia.rohani@sbmu.ac.ir
\end{abstract}

How to cite this paper: Hasan Shiri, F., Mohtashami, J., Manoochehri, H., Nasiri, M. and Rohani, C. (2020) Care Plan for Reducing Stigma in Cancer Disease. Open Journal of Nursing, 10, 1142-1154. https://doi.org/10.4236/ojn.2020.1011081

Received: October 26, 2020

Accepted: November 27, 2020

Published: November 30, 2020

Copyright (c) 2020 by author(s) and Scientific Research Publishing Inc. This work is licensed under the Creative Commons Attribution International License (CC BY 4.0).

http://creativecommons.org/licenses/by/4.0/

\section{(c) (i) Open Access}

\begin{abstract}
Purpose: Disease-related Stigma is negative emotions, attitudes, stereotypes, and beliefs about diseases. Cancer is one of the diseases that can be exposed to stigma. Regarding the effects of stigma on the quality of life; admission and adherence to treatment, and considering stigma as a barrier to health promotion and cancer screening, the aim of this study was to provide a care plan for reducing stigma in cancer. Methods: This research was carried out during three phases of qualitative, quantitative studies and a review of the literature. The qualitative phase was conducted by conventional content analysis to find effective factors on the reduction of stigma through face to face semi-structured interviews. The quantitative phase was a cross-sectional descriptive study to measure the level of stigma. By using both quantitative and qualitative findings, and a review of the literature, a comprehensive care plan to reduce stigma in families with cancer patients was developed. Results: The results showed two categories of data; 1) reducing stigma at the individual or family level which needs increasing awareness, teaching coping skills, and support, as well as counseling; and 2) stigma reduction at the community level and policy rules which involve public education and cultural changes. Conclusion: Implementation of a care plan for reducing stigma can increase the physical and psychological health outcomes of people influenced by cancer, and at the community level, improves the attitude toward cancer and the success of screening programs, and ultimately reduces disability and mortality of the disease.
\end{abstract}

\section{Keywords}

Stigma, Cancer, Stigma Control, Care Plan 


\section{Background}

Disease-related stigma is a set of negative attitudes, beliefs, thoughts and behaviors towards someone with a chronic disease or obvious physical disability [1] [2] [3]. Nielsen describes Stigma as a social disparity and the main elements of Stigma as "labeling, stereotypy, separation, loss of position, and discrimination", which affects the integrity of the individual [4] [5]. Data from stigma suggest that cancer is one of the diseases that have a high stigma in many societies [2] [6]. Cancer is usually considered a life-threatening illness and is synonymous with suffering and death [7] [8]. The image of death, bad fate, disaster and misery are some parts of attitude toward cancer [9]. In addition to the human health threat, cancer may be a deviation from the norm and an undesirable quality and the affected person can be exposed to negative emotions of others [5] [10].

The process of stigmatization usually begins with the diagnosis of the disease [4]. Stigma causes psychosocial problems that affect all aspects of the individual's life and interfere with the treatment of disease [11]. Even treatment may also lead to stigma and disrupt interpersonal relationships [12]. Cancer can lead to changes in individual identity, experiencing emotional distresses, social dysfunction, physical and emotional consequences of appearance changes, social stigma and marginality in the community [12]. The fear of cancer stigma and the perception of the disease, shame and anxiety of having cancer, prevent seeking health services [6] [12]. In Asian countries, some patients travel to other countries for treatment because they want to keep their disease a secret from neighbors, friends and/or family, and since the treatment is very expensive in those countries, it can lead to leaving of treatment in people who are not financially capable [13].

Stigma is a widespread and deep cultural concept, the effects, dimensions and consequences of which in the local context are significant. It affects individuals, families and even the effectiveness of public health programs [12] [14]. Therefore, it is important to address the methods that can reduce stigma or its effects on affected people.

Considering the limited quantitative and qualitative studies on the stigma in cancer in Iran, and that people with cancer and their families are suffering from stigma and its psychological and social problems, this study aimed to provide a "care plan for reducing stigma in cancer" disease. Many articles in other parts of the world have proposed a number of strategies to reduce stigma, some of them have been tested in cancer. However, there is not a comprehensive caring plan for using in this group, so this care plan can be considered as a package that is usable in a clinical setting to help reduce stigma and its consequences.

\section{Methods}

The present research was approved by the Ethics Committee of Shahid Beheshti University of Medical Sciences under the code of IRSBMU.PHNM1395-570. To achieve a care plan for reducing stigma in cancer, three phases were carried out. 
First, a qualitative study was conducted to explain the nature of the disease and the experience of people affected by the cancer in coping with stigma by conventional content analysis. Qualitative phase was conducted through content analysis by face to face interview. Before the interview, the participants agreed with the researcher on the time and place of the interview. The interview location was a private and quiet place in hospital or office of the participants with the pre consent. The research area included the affiliated centers of Shahid Beheshti University of Medical Sciences, including the Cancer Research Center, Shohaday Tajrish Hospital, Imam Hossein Hospital and Kashan Shahid Beheshti Hospital affiliated to Kashan University of Medical Sciences. The participants were selected through purposive sampling. In this method, the researcher was looking for people who had a rich experience in the subject and were able to express this experience. Contributors included cancer patients, home caregivers, and health care providers. Interviews were performed until data reached the saturation. A telephone interview was conducted on one of the interviews to complete and resolve the ambiguity after the interview was completed. The average interview time was 25 minutes. The data were collected through semi-structured interviews. The focus of the interview questions was the experiences of cancer patients who initially asked a general question "Cancer created what changes in your life and communications?" "What changes have you seen in the lives of people with cancer and their associations, and what is your experience with cancer stigma?" The rest of the questions were used based on the participants' answers. Samples were purposefully selected from medical staff, patients and home caregivers of cancer patients. The location of the interview was agreed upon and 14 interviews were switched to data saturation. Qualitative analysis was performed on the data.

In the next step, a quantitative research by descriptive method was conducted to measure the amount of stigma of the cancer and related factors in individuals with cancer which was published elsewhere [15] [16]. After reviewing the qualitative and quantitative results, a literature reviewin order to find reducing stigma methods in diseases and in particular cancer was also carried out. Articles and dissertations in PubMed, Scopus, Science Direct, ProQuest, Google Scholar databases, as well as Iranmedex, Iran doc and SID Persian databases with a combination of Persian and English keywords "stigma, tag, Shame, discrimination" "strategy, method, plan, fight, Combat, overcome and reduce, Guideline", "Health, disease, cancer" were searched. The study search flowchart is in Diagram 1. In this way all of 61 full-text articles related to the stigma and methods to overcome it in different diseases, especially cancer, including quantitative, qualitative, review articles, dissertations and clinical guidelines were reviewed (Figure 1).

\section{Results}

Due to the data analysis in qualitative part, two main themes were obtained: considering the cancer as a threatening and unpleasant disease (with subthemes: 
fear and concern, the undisputed destiny cultural superstition and stereotypes), and conflict of identity/psychosocial disruption (sub themes included: communicative disruption, concealment of disease, psychological reactions disturbance in body image, conflict of doubt and certainty). The findings of quantitative part also showed that $25.2 \%$ of the subjects had high score of the stigma. The results of logistic regression and the relationship between the severity of stigma with demographic variables and clinical history indicated that the stigma was related to the level of education of the participants. Increasing the level of education was associated with a reduction in the score of the stigma. After combination of the results of qualitative and quantitative and literature review, a "care plan to reduce stigma in cancer" in two levels was designed: 1) focused on people with cancer/their family; 2) focused on the community/policy (Figure 2).

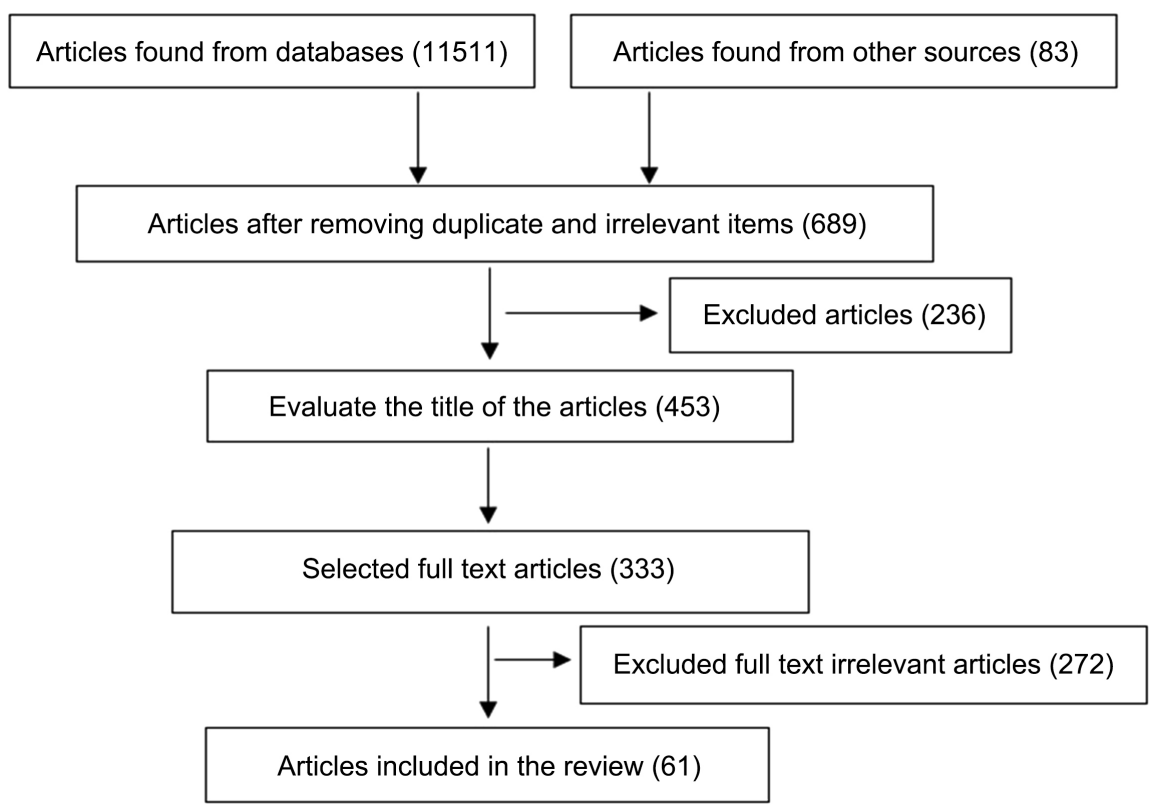

Figure 1. Study search flowchart.

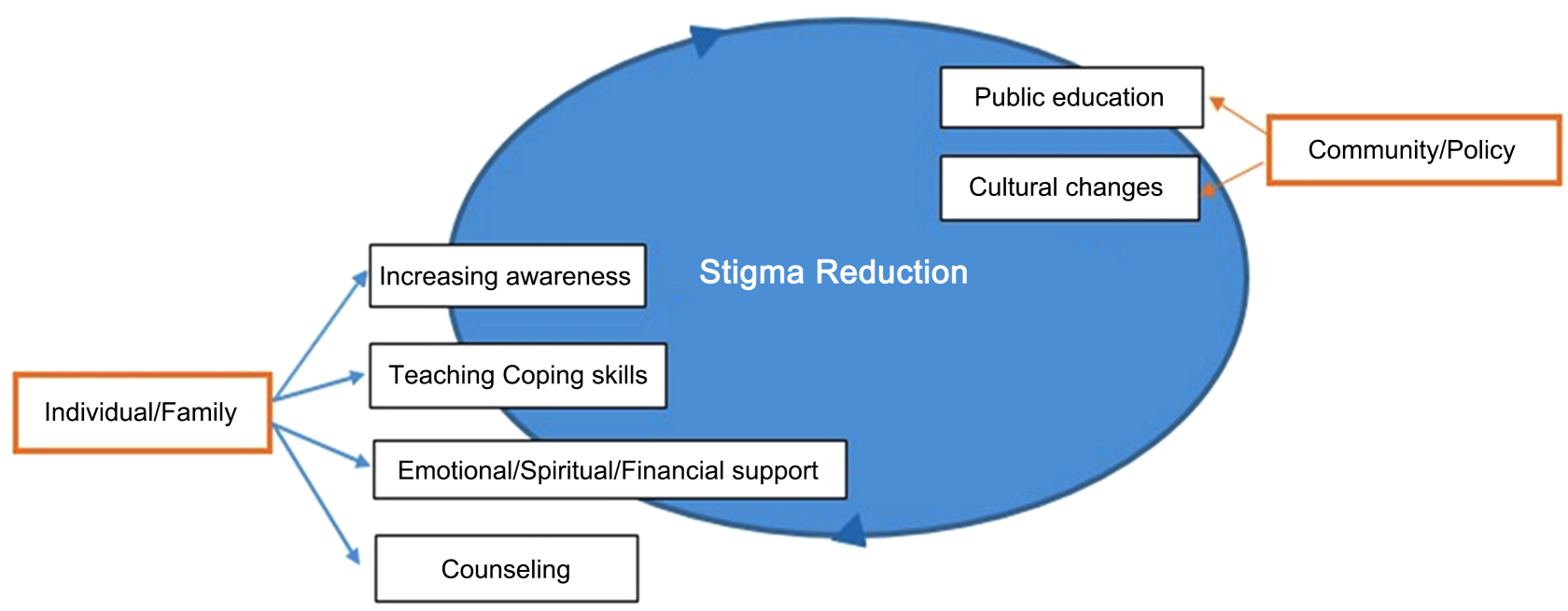

Figure 2. Care plan for reducing stigma. 


\subsection{Individual and Family Level}

\subsubsection{Increasing Awareness}

Frequent assessment of interviewing data showed that there is not enough information about the disease on the level of the community as well as patients and their relatives, which causes confusion and doubt in the decision-making process, as well as internalizing the wrong beliefs and behaviors of others. Giving information to the patient/family about the disease, treatment steps and possible complications, mentioning the possible causes of the disease and the factors affecting the incidence of cancer in order to eliminate false beliefs such as (bad fate, evil eye, punishment of God), the scientific reasons to apply communication constraints in the course of chemotherapy, radiation therapy, and the reduction of the activity of the immune system (if necessary); explain the temporary effects of some complications such as mouth ulcers, digestive complications, nausea, vomiting, hair loss, which is some information that if known, can reduce the amount of stigma in the individual and family, and reduce the impact of social stigma on the affected people.

\subsubsection{Teaching Coping Skills}

The results showed that one of the effective strategies for reducing stigma is the use of coping skills by the affected person/family in dealing with negative attitudes and stigma behavior of others. Perform affairs such as reading books, handicrafts, creative activities, and so on; identifying support sources such as family, friends, or acquaintances, accepting assistance and getting support from them; doing spiritual activities and religious activities (according to Interests and personal beliefs), spiritual interventions such as prayer, worship; relaxation techniques such as deep breathing, guiding imagery and so on; ways to cope with attitudes, behaviors and judgment of others such as neglect, expressing positive opinions, expressing hope and giving right information to others help to increase personal growth and the fight against stigma.

\subsubsection{Emotional/Spiritual/Financial Support}

In this part of the plan, the role of the family and especially the spouse has been emphasized in reducing the stigma. Meanwhile, the support provided by the medical staff, especially the physician and nurse, has been emphasized on the reduction of stigma. Some aspects of emotional support, mentioned in this plan, were accessibility, communication and maintaining it, counseling centers and supportive societies. Also maintaining the routine and favorite communication; membership of support and peer groups is another help. Assessing problems and financial constraints and introducing person and family to a social worker, support sources, support groups, charities and sponsoring organizations is another part of emotional, financial and instrumental support.

\subsubsection{Counseling}

Most participants emphasized the need for counseling in the early diagnosis pe- 
riod and prior to the onset of treatment for the affected person/family. Also, in case of severe grief and sadness and severe reactions to the diagnosis, counseling is an essential part that can eliminate the psychological disturbance as well as negative attitudes, and create hope and positive belief in treatment, resulting in raises in survival rate by continuing the treatment.

\subsection{Community Level and Policy}

\subsubsection{Public Education}

The results indicate that the stigma is dependent on cultural stereotypes and community awareness level. Education about initial warning symptom, the importance of screening for early detection of cancer to prevent unnecessary fear of cancer, self-care behaviors such as good nutrition, exercise, healthy lifestyle and preventing stress and high-risk behaviors can lead to an increase in people's attention to warning signs. Also, teaching how to deal with person with cancer, their needs, desires, and how to communicate with them helps to improve the attitudes and behavior of others towards the patients and reduce the sense of stigma in these individuals.

\subsubsection{Cultural Change}

The results suggest that cultural changes are needed to reduce wrong beliefs about cancer, and negative attitudes and behaviors toward affected people. Possible actions include: educational and cultural billboards in public places, posters and educational leaflets, development of screening tests focusing on reducing stigmatization and willingness to do tests. Also, scientific programs, congresses, supportive associations and educational institutions; publication of the success stories of cancer survivors in books, magazines and internet to develop positive attitudes and reduce fear; develop programs with participation of cancer scientists and professionals are other actions that help enhance cultural belief.

\section{Discussion}

This study was designed and implemented in order to achieve a care plan for reducing stigma in cancer. The most important issues that require interventions in the care plan are discussed in this section, respectively.

\subsection{Increasing Awareness}

Based on the results, increasing awareness and information is one of the effective strategies for reducing individual and social stigma in affected people and their families. The results showed that lack of sufficient information on the disease, the treatment process, and possible complications and managing them, as well as the nature of cancer and the consequences of treatment complications, cause fears and concerns about cancer and negative public perceptions of the community. Misconceptions and psychosocial factors in people with chronic illness are the most important causes of stigma. Insufficient knowledge about the disease affects people's attitudes and can exacerbate stigma [17]. Many people with can- 
cer speak about lack of information associated with the side effects of treatments during and after disease [18] [19]. Providing information on whether cancer can be controlled even if it cannot be healed is an important strategy in reducing fear and stigma [20]. Knowledge is a good tool for reducing the anxiety caused by disease stigma, negative or undesirable feelings, or feelings of guilt and embarrassment [21]. Education can help people fight fear and complications of the disease and remove the burden of stigma [18].

\subsection{Coping Skills}

One of the results in this study was the use of coping skills for individual or group empowerment, which were among the effective strategies for reducing stigma among the participants. Various studies have shown the success of applying coping strategies to counteract situations at individual or group level. Care, support, and counseling strategies are interventions aimed at empowering an individual or group of affected or injured persons to combat stigma [22]. Accepting the diagnosis of disease and maintaining hope along with social support will inhibit self-stigma due to diagnosis, and it can be said that accepting the disease is a key element in engaging in care because it will be possible to follow treatment plan, provide courage to face cancer and believe there is a chance to survive [23]. For many participants in the Meacham study, the acceptance of the disease was driven by their religious belief in fate and, using these coping techniques, participants could start medical treatment, despite social pressures to follow up on herbal treatment options [24]. Potential coping mechanisms in African-American women with breast cancer include spirituality, positive attitude and supportive networks that have had a positive impact on the reconciliation of their lives. Believing in God and the power of worship as the most important part of faith, an important coping mechanism and a vital facilitator of the chance of survival are mentioned in Torres et al. 2016. Maintaining the routines of everyday life, communication and naturalization strategies are considered as positive coping mechanisms [5] [25]. Faith and belief in God, the power of worship, positive attitudes and empowerment such as hobbies, fun, cheerful spirit, and the development of individual skills are among the set of strategies that help to overcome stigma and enhance self-efficacy and personal worth [19] [22]. Membership in support groups, where individuals can share their experiences and learn coping skills or support peers is another effective aid in reducing stigma [26].

\subsection{Support}

While social support in cancer care is not well-defined, it plays an important role in stabilizing the threat to individual identities [21]. Patients seek emotional and informational support from family, friends, and health care providers throughout their lives with disease [27]. Lack of strong social support and cancer knowledge is one of the most influential factors on stigma [23]. Social support is one 
of the most important determinants of the response to the disease. It can reduce the severity of the response to disease related stressors [28]. Support is one of the most effective factors in social adjustment, improving physical health and well-being, and reducing anxiety and depression after severe physical illnesses. Social support in cancer is recognized as an interactive institution, including the transmission of emotions, concerns, services and information. However, people with cancer may have problems in obtaining adequate support and are at risk of losing the benefits of social relationships, and even social relationships, rather than support, in some cases, are another source of emotional anxiety for these people [5]. As Jarret (2015) states, many cancer patients have unmet needs for care, such as socio-emotional and physical support. Maintaining these needs can lead to serious physiological symptoms and psychological distress [29]. The results of this study also indicate that affected people need comprehensive support from the family and especially their spouses. Many women argue that the quality of emotional support from several key sources, including husbands, close friends and family, is more important than having a large number of supporters. The results indicate that emotional support behaviors are very useful to those who have life-threatening illnesses like cancer, with words of love, care, concern, understanding and affection [5]. Many participants in the study also talked about lack of support and communication deficits between themselves and the doctors. Many women with breast cancer experience emotional disturbances; social dysfunction due to treatment and social stigma associated with the disease, and are forced to manage their own stigma and problems without adequate professional and social support [1].

\subsection{Counseling}

One of the purposes of other articles and our qualitative study was to provide counseling and receive professional help for psychological strengthening and reduction of stigma in affected people and their family members. Stigma and negative or undesirable feelings or feelings of guilt and embarrassment can cause more stress and psychological and social complications in people with disease [21]. Stigma is a major barrier to the diagnosis of psychological distress and use of psychosocial services among cancer patients [30]. One of the points mentioned in the study and shown in the evidence of other studies is the need for counseling for patients, especially in the early stages after diagnosis. The approach to providing information along with counseling, as well as psychosocial interventions and counseling after testing and stress management programs, are considered as measures for HIV/AIDS-affected people [24]. Providing counseling to assist patients in overcoming cancer, along with the development of advanced and early diagnostic treatments and the elimination of fear of death are part of a series of measures in the field of psycho-oncology [19]. In summary and as the results show, psychological reinforcement along with family support and palliative, spiritual, and religious help, and the membership of individuals in 
support groups or peers group leads to a reduction in stigma and a feeling of isolation [5].

\subsection{Public Education and Cultural Change}

Stigma has two effects: firstly, the presence of stigma causes other people to react negatively to those who are affected, which affects the psychological health of the patients to follow the treatment and return to normal life after cancer [6]. Secondly, it prevents people from learning about warning signs and receiving professional services [31]. Because, as pointed out, Stigma is a social cultural concept that varies between cultures and over time and is influenced by social beliefs, gender, age, religion, and membership in social groups [1] [3] [32] [33] [34]. The social experiences of women and their beliefs point to untreatable and severe fear of cancer, and the fear of cancer stigma and the tragic nature of the disease delay the search for medical assistance [31]. Researchers suggest one of the main causes of delay in referring is social stigma of cancer, economic pressures and other organizational barriers [23] [35]. Cultural and educational efforts can increase awareness and reduce the stigma of cancer [27]. Ramacant $e t$ al. have talked about raising awareness of breast cancer through mass media and television, street camps and colleges, along with group discussions and debates [35]. Expressing successes in cancer through media by providing information on issues such as reducing the incidence of cancer (where applicable), promoting screening and early detection programs, treatment approaches, palliative care, survivors of cancer, governmental efforts in controlling and preventing cancer, new research, or cancer-related investments and operations, reduce the stigma associated with cancer [20]. One way to reduce fear is to increase the role of cancer survivors and supportive programs. If more people hear the stories of the survivors, cancer will be less associated with fear and death [23]. General education has been effective in modifying traditional beliefs and attitudes toward disease and reducing stigma and discrimination towards patients [22]. Media is expected to play an effective role in raising public awareness to control the disease and reduce stigma, but the participants acknowledged that the role of the media in reducing stigma is limited, and even the negative image of cancer through the media was seen in description of participants that develop stigma. Mentioning precise statistics of cancer can sometimes be fearful, but at the same time making progress on cancer through media coverage reduces stigma associated with cancer [20] [36]. The results of the study by Mardani-Hamooleh and Heidarisuggest that cultural and educational efforts can increase awareness and reduce the stigma of cancer [27]. In this context, programs such as creating media campaigns aimed at trying to change people's beliefs are effective [13].

Meacham (2016) revealed that extensive screening campaigns are needed to improve screening behaviors, which can be enhanced by describing survivors' stories. These campaigns should be available for all areas of the country and there should be training for all age and sex groups such as children, men and 
women [23]. (Meacham, 2015). This education on cancer helps improve attitudes in people [37]. Asadzadeh et al. (2011) considered awareness raising via general education in society as one of the ways to overcome social misconceptions about the cause of breast cancer and improve screening behaviors in Iran [38]. Also, there is an emphasis that health education and prevention campaigns should provide information about behavioral risks to cancer in a way that more people (in relation to cancer or those doing so) do not suffer from stigma [30].

\subsection{Applications}

Stigma Reduction Care Plan can help caregiver to identify stigma and using its strategies reduce negative effects of stigma and improve quality of individual, familial, spiritual and social life of affected people. According to the results of the present study, "Stigma Reduction Care Plan" can help caregivers in the clinic to plan and do appropriate action based on patient and family circumstances. In the first step, nurses should be made aware of this phenomenon because stigma is seen even in care of patients and affects the quality of care and client satisfaction. If caregivers know this concept, they can diagnose the signs and symptoms of stigma in people affected by the disease and take appropriate care. According to the research findings, increasing health awareness and knowledge is one of the essential needs in reducing stigma for both patients and the family and the general population; It is also important to change attitudes and eliminate cultural stereotypes in this regard, which should be considered in health education programs.

\section{Conclusion}

With regard to cancer-related stigma, interventions aimed at reducing fear of cancer help reduce stigma and promote participation in cancer care. In addition, the results indicate that individual empowerment, support and counseling are other ways to overcome the stigma and reduce its effects in cancer-affected individuals. At the community level, information and cultural changes are also advocated in addressing the negative attitudes toward cancer and patients, and the success of screening and control programs. The implementation of this care plan can help care providers manage and control some parts of the psychosocial problems of affected people and their families.

\section{Acknowledgments}

We thank all participants in this study, faculty members of Shahid Beheshti University of Medical Sciences, School of Nursing and Midwifery, Dr. Maryam Rasouli, Parvaneh-vasli, also Dr. Nahid Reje, Dr. Mansoureh Ashghali Farahani, respected personnel of Oncology Departments of university hospitals; ShohadaTajrish, and Imam Hossein (AS) in Tehran, and Shahid Beheshti hospital of Kashan. This article is a result of the Ph.D. thesis in Nursing, approved by the Research Committee of Shahid Beheshti University of Medical Sciences. 


\section{Conflicts of Interest}

The authors declare no conflicts of interest regarding the publication of this paper.

\section{References}

[1] Suwankhong, D. and Liamputtong, P. (2016) Breast Cancer Treatment Experiences of Changes and Social Stigma among Thai Women in Southern Thailand. Cancer Nursing, 39, 213-220. https://doi.org/10.1097/NCC.0000000000000255

[2] Edelen, M.O., Chandra, A., Stucky, B., Schear, R., Neal, C. and Rechis, R. (2014) Developing a Global Cancer Stigma Index. SAGE Open, 4, 1-9.

https://doi.org/10.1177/2158244014547875

[3] Marlow, L.A.V. and Wardle, J. (2014) Development of a Scale to Assess Cancer Stigma in the Non-Patient Population. BMC Cancer, 14, 285.

https://doi.org/10.1186/1471-2407-14-285

[4] Dubin, R.E., Kaplan, A., Graves, L.K. and Ng, V. (2017) Acknowledging Stigma Its Presence in Patient Care and Medical Education. Canadian Family Physician, 63, 906-908.

[5] Flanagan, J. and Holmes, S. (2001) Social Perceptions of Cancer and Their Impacts: Implications for Nursing Practice Arising from the Literature. Journal of Advanced Nursing, 32, 740-749. https://doi.org/10.1046/j.1365-2648.2000.01535.x

[6] Fujisawa, D. and Hagiwara, N. (2015) Cancer Stigma and Its Health Consequences. Current Breast Cancer Reports, 7, 143-150.

https://doi.org/10.1007/s12609-015-0185-0

[7] Ehsani, M., Taleghani, F., Hematti, S. and Abazari, P. (2016) Perceptions of Patients, Families, Physicians and Nurses Regarding Challenges in Cancer Disclosure: A Descriptive Qualitative Study. European Journal of Oncology Nursing, 25, 55-61. https://doi.org/10.1016/j.ejon.2016.09.003

[8] Sette, C.P., Capitão, C.G. and Carvalho, D.F.L. (2016) Depressive Symptoms in Patients with Cancer. Open Journal of Medical Psychology, 5, 7-16.

https://doi.org/10.4236/ojmp.2016.51002

[9] Wilson, K. and Luker, K.A. (2006) At Home in Hospital? Interaction and Stigma in People Affected by Cancer. Social Science \& Medicine, 62, 1616-1627. https://doi.org/10.1016/j.socscimed.2005.08.053

[10] Mohabbat-Bahar, S., Bigdeli, I., Mashhadi, A. and Moradi-Joo, M. (2017) Investigation of Stigma Phenomenon in Cancer: A Grounded Theory Study. Iranian Journal of Cancer Prevention, 10, e6596. https://doi.org/10.17795/ijcp-6596

[11] Akbari, M.E. (2010) Cancer and Emotion; the Scope of Psycho-Socio-Oncology. Iranian Journal of Cancer Prevention, 3, e80661.

[12] Tang, P.-L., Mayer, D.K., Chou, F.-H. and Hsiao, K.-Y. (2015) The Experience of Cancer Stigma in Taiwan: A Qualitative Study of Female Cancer Patients. Archives of Psychiatric Nursing, 30, 204-209. http://www.elsevier.com/locate/apnu https://doi.org/10.1016/j.apnu.2015.08.015

[13] Al-Azri, M., Al-Awisi, H., Al-Rasbi, S., El-Shafie, K., Al-Hinai, M., Al-Habsi, H. and Al-Moundhri, M. (2014) Psychosocial Impact of Breast Cancer Diagnosis among Omani Women. Oman Medical Journal, 29, 437-444. https://doi.org/10.5001/omj.2014.115

[14] Yllmaz, M., Dissiz, G., Usluoglu, A.K., Iriz, S., Demir, F., and Alacacioglu, A.(2020) 
Cancer-Related Stigma and Depression in Cancer Patients in A Middle-Income Country. Asia-Pacific Journal of Oncology Nursing, 7, 95-102. https://doi.org/10.4103/apjon.apjon_45_19

[15] Hasan Shiri, F., Mohtashami, J., Manoochehri, H. and Rohan, I.C. (2018) Explaining the Meaning of Cancer Stigma from the Point of View of Iranian Stakeholders: A Qualitative Study. International Journal of Cancer Management, 11, e61165. https://doi.org/10.5812/ijcm.61165

[16] Hasan Shiri, F., Mohtashami, J., Nasiri, M., Manoochehri, H. and Rohani, C. (2018) Stigma and Related Factors in Iranian People with Cancer. Asian Pacific Journal of Cancer Prevention, 19, 2285-2290.

[17] Dogan, A.P., Atakli, D., Yuksel, B., Tekin Guveli, B. and Sari, H. (2015) Stigmatization and Social Impacts of Epilepsy in Turkey. Epilepsy \& Behavior, 50, 50-54. https://doi.org/10.1016/j.yebeh.2015.05.014

[18] Cognetti, J.M. (2013) Perceptions of Breast Cancer-Related Stigma and Genetic Knowledge among Latina Women. Degree of Master of Science Dissertation, School of Medicine, University of South Carolina, Columbia. https://scholarcommons.sc.edu/etd/1251

[19] Torres, E., Dixon, C. and Richman, A.R. (2016) Understanding the Breast Cancer Experience of Survivors: A Qualitative Study of African American Women in Rural Eastern North Carolina. Journal of Cancer Education, 31, 198-206. https://doi.org/10.1007/s13187-015-0833-0

[20] Neal, C., Beckjord, E.B., Rechis, R., Schaeffer, J., Berno, D. and Duchover, Y. (2010) Cancer Stigma and Silence around the World: A Livestrong Report. http://www.livestrong.org/What-We-Do/Our-Approach/Reports-Findings/CancerStigma-and-Silence-Around-the-World

[21] Molem, V.D. (1999) Relating Information Needs to the Cancer Experience: Information as a Key Coping Strategy. European Journal of Cancer Care, 8, 238-244. https://doi.org/10.1046/j.1365-2354.1999.00176.x

[22] Heijnders, M. and Meij, S.V.D. (2006) The Fight against Stigma: An Overview of Stigma-Reduction Strategies and Interventions. Psychology, Health \& Medicine, 11, 353-363. https://doi.org/10.1080/13548500600595327

[23] Meacham, E.G. (2015) Exploring Stigma as a Barrier to Cancer Service Engagement: Illness Narratives of Breast Cancer Survivors in Kampala, Uganda. Degree of Master of Science Dissertation of Public Health, University of Washington, Seattle.

[24] Stewart, D.E., Cheung, A.M., Duff, S., Wong, F., McQuestion, M., Cheng, T., Purdy, L. and Bunston, T. (2001) Attributions of Cause and Recurrence in Long-Term Breast Cancer Survivors. Psycho-Oncology, 10, 179-183.

https://doi.org/10.1002/pon.497

[25] Oates, E.D. (1984) Fighting Cancer, Dying to Win: The American Strategy for Creating a Chronic Sick Role (Illness Behavior, Doctor-Patient Relationship, Stigma, Western Biomedicine). Southern Methodist University, Dallas, Number: 8507787.

[26] Koschorke, M., Padmavati, R., Kumar, S., Cohen, A., Weiss, H.A., Chatterjee, S., Pereira, J., Naik, S., John, S., Dabholkar, H., Balaji, M., Chavan, A., Varghese, M., Thara, R., Thornicroft, G. and Patel, V. (2014) Experiences of Stigma and Discrimination of People with Schizophrenia in India. Social Science \& Medicine, 123, 149-159. http://www.elsevier.com/locate/socscimed https://doi.org/10.1016/j.socscimed.2014.10.035

[27] Mardani-Hamooleh, M. and Heidari, H. (2016)Cancer Patients' Effort to Return to Normal Life: A Hermeneutic Study. Nordic College of Caring Science, Oslo, 1-8. 
https://doi.org/10.1111/scs.12354

[28] Schwarzer, R. and Weiner, B. (1991) Stigma Controllability and Coping as Predictors of Emotions and Social Support. Journal of Social and Personal Relationships, 8, 133-140. https://doi.org/10.1177/0265407591081007

[29] Jarrett, L.A. (2015) Health-Related Stigma in Advanced Lung Cancer. PhD Dissertation, Faculty of the Graduate School of Vanderbilt University, Nashville.

[30] Holland, J.C. (2010) Why Psychosocial Care Is Difficult to Integrate into Routine Cancer Care: Stigma Is the Elephant in the Room. Journal of the National Comprehensive Cancer Network, 8, 362-366. https://doi.org/10.6004/jnccn.2010.0028

[31] Khakbazan, Z., LatifnejadRoudsari, R. and Mohammadi, E. (2014) Help Seeking Behavior of Women with Self-Discovered Breast Cancer Symptoms: A Meta-Ethnographic Synthesis of Patient Delay. PLoS ONE, 9, e110262. https://doi.org/10.1371/journal.pone.0110262

[32] Bracke, P., Delaruelle, K. and Verhaeghe, M. (2019) Dominant Cultural and Personal Stigma Beliefs and the Utilization of Mental Health Services: A Cross-National Comparison. Frontiers in Sociology, 4, 1-12.

https://doi.org/10.3389/fsoc.2019.00040

[33] Kim, M.A. (2012) The Effects of Physical Functioning and Public Stigma on Psychological Distress as Mediated by Cognitive and Social Factors among Korean Survivors of Childhood Cancer. PhD Dissertation, University of Southern California, Los Angeles.

[34] Anderson, N., Andrews, M., Bent, K.N., Douglas, M.K., Elhammoumi, Ch.V., Keenan, C., Kemppainen, J.K., Lipson, J.G., Thompson, M.C. and Mattson, S. (2010) Culturally Based Health and Illness Beliefs and Practices across the Life Span. Journal of Transcultural Nursing, 21, 152S-235S. https://doi.org/10.1177/1043659610381094

[35] Ramakant, P., Singh K.H., Jaiswal, S., Singh, S., Ranjan, P., Rana, C., Jain, V. and Mishra, A.K. (2017) A Survey on Breast Cancer Awareness among Medical, Paramedical, and General Population in North India Using Self-Designed Questionnaire: A Prospective Study. Indian Journal of Surgical Oncology, 9, 323-327. https://doi.org/10.1007/s13193-017-0703-9

[36] Yeshua-Katz, D., Shvarts, S.H. and Segal-Engelchin, D. (2019) Hierarchy of Hair Loss Stigma: Media Portrayals of Cancer, Alopecia Areata, and Ringworm in Israeli Newspapers. Israel Journal of Health Policy Research, 8, Article No. 68. https://doi.org/10.1186/s13584-019-0338-0

[37] Criswell, K.R. (2016) Lung Cancer Stigma: Associated Variables and Coping Strategies. PhD Dissertation, Doctor of Philosophy.

http://scholarsrepository.llu.edu/etd/330

[38] Asadzadeh, V.F., Broeders, M.J.M., Kiemeney, L.A.L.M. and Verbeek, A.L.M. (2011) Opportunity for Breast Cancer Screening in Limited Resource Countries: A Literature Review and Implications for Iran. Asian Pacific Journal of Cancer Prevention, 12, 2467-2475. http://www.ncbi.nlm.nih.gov/pubmed/22320940 